\title{
CORRIGENDUM
}

\section{Dietary fibre and fermentability characteristics of root crops and legumes}

Aida C. Mallillin, Trinidad P. Trinidad, Ruby Raterta, Kevin Dagbay and Anacleta S. Loyola doi:10.1017/S000711450891151X, Published by Cambridge University Press, 11 March, 2008.

(First published online 5 June 2008)

In error, some information given in table 1 was incorrectly published. The correct version can be found below.

Table 1. Nutritional composition of root crops and legumes ( $\mathrm{g} / 100 \mathrm{~g}$ sample)

(Mean values with their standard errors)

\begin{tabular}{|c|c|c|c|c|c|c|c|c|c|c|c|c|c|c|c|c|c|c|}
\hline \multirow[b]{2}{*}{ Test foods } & \multicolumn{2}{|c|}{ Fat } & \multicolumn{2}{|c|}{ Protein } & \multicolumn{2}{|c|}{$\mathrm{CHO}^{*}$} & \multicolumn{2}{|c|}{$\begin{array}{l}\text { Dietary } \\
\text { fibre }\end{array}$} & \multicolumn{2}{|c|}{$\begin{array}{l}\text { Soluble } \\
\text { fibre }\end{array}$} & \multicolumn{2}{|c|}{$\begin{array}{l}\text { Insoluble } \\
\text { fibre }\end{array}$} & \multicolumn{2}{|c|}{ Acetate† } & \multicolumn{2}{|c|}{ Propionate† } & \multicolumn{2}{|c|}{ Butyrate† } \\
\hline & Mean & SEM & Mean & SEM & Mean & SEM & Mean & SEM & Mean & SEM & Mean & SEM & Mean & SEM & Mean & SEM & Mean & SEM \\
\hline \multicolumn{19}{|l|}{ Root crops } \\
\hline Kamote & $0.6^{i}$ & 0.2 & $3 \cdot 4^{h}$ & 0.0 & $89 \cdot 6^{c}$ & 0.3 & $8 \cdot 1^{j}$ & 0.1 & $4 \cdot 8^{\mathrm{C}}$ & 0.4 & $3 \cdot 2^{i}$ & 0.2 & $1.1^{9 x}$ & 0.2 & $0.4^{\mathrm{hz}}$ & 0.0 & $0.5^{\mathrm{jy}}$ & 0.0 \\
\hline Gabi & $0.4^{i}$ & 0.0 & $1.1^{j}$ & 0.0 & $90 \cdot 7^{b}$ & 0.2 & $13.5^{9}$ & 0.1 & $3.6^{\mathrm{e}}$ & 0.1 & $9.5^{f}$ & 0.2 & $1.1^{9 x}$ & 0.1 & $0.2^{\mathrm{iy}}$ & 0.0 & $0.2^{\mathrm{ky}}$ & 0.0 \\
\hline Potato & $1.0^{\mathrm{k}}$ & 0.1 & $10 \cdot 0^{\mathrm{e}}$ & 0.1 & $77 \cdot 1^{\mathrm{e}}$ & 0.4 & $7 \cdot 6^{\mathrm{k}}$ & 0.2 & $3.6^{\mathrm{e}}$ & 0.1 & $4 \cdot 0^{\mathrm{h}}$ & 0.2 & $0.2^{\text {hy }}$ & 0.0 & $0.1^{\mathrm{jy}}$ & 0.1 & $0.6^{\text {hijx }}$ & 0.1 \\
\hline Tugi & $0.2^{\mathrm{jk}}$ & 0.1 & $4 \cdot 8^{\mathrm{g}}$ & 0.1 & $75 \cdot 6^{f}$ & 0.8 & $10 \cdot 3^{i}$ & 0.1 & $3 \cdot 6^{\mathrm{e}}$ & 0.1 & $7.0^{9}$ & 0.2 & $2 \cdot 5^{\mathrm{dex}}$ & 0.4 & $1 \cdot 8^{\text {dey }}$ & 0.2 & $0.8^{\mathrm{gz}}$ & 0.0 \\
\hline Ube & $0.4^{i}$ & 0.0 & $5 \cdot 2^{f}$ & 0.1 & $87 \cdot 0^{\mathrm{d}}$ & 0.3 & $11 \cdot 8^{\mathrm{h}}$ & 0.2 & $4 \cdot 4^{\mathrm{d}}$ & $0 \cdot 1$ & $7 \cdot 4^{9}$ & 0.2 & $0.2^{\mathrm{hx}}$ & 0.0 & $0.1^{\mathrm{yy}}$ & 0.0 & $0.1^{\mathrm{kx}}$ & 0.1 \\
\hline Cassava & $0.3^{\mathrm{j}}$ & 0.0 & $2 \cdot 4^{i}$ & 0.0 & $91 \cdot 1^{a}$ & 0.1 & $4 \cdot 6^{1}$ & 0.2 & $1.4^{\mathrm{g}}$ & 0.4 & $3 \cdot 2^{i}$ & 0.1 & $2 \cdot 4^{\mathrm{ex}}$ & 0.1 & $0.7^{\mathrm{tz}}$ & 0.1 & $0.8^{g x}$ & 0.0 \\
\hline \multicolumn{19}{|l|}{ Legumes } \\
\hline Mungbean & $5 \cdot 8^{\mathrm{b}}$ & 0.4 & $14.6^{\text {de }}$ & 4.9 & $64 \cdot 1^{\mathrm{gh}}$ & 5.4 & $31 \cdot 7^{\mathrm{c}}$ & 0.1 & $4 \cdot 8^{\mathrm{c}}$ & 0.3 & $26 \cdot 9^{c}$ & 0.2 & $5 \cdot 3^{\mathrm{ax}}$ & 0.1 & $1 \cdot 7^{\mathrm{dz}}$ & 0.0 & $2 \cdot 5^{\mathrm{dy}}$ & 0.2 \\
\hline Soyabean & $2 \cdot 8^{\mathrm{e}}$ & 0.1 & $33 \cdot 3^{\mathrm{a}}$ & 1.8 & $55 \cdot 8^{i}$ & 1.9 & $46 \cdot 9^{a}$ & 3.4 & $8.0^{\mathrm{a}}$ & 0.2 & $38.9^{\mathrm{a}}$ & 0.2 & $3 \cdot 8^{\mathrm{cx}}$ & 0.5 & $1.4^{\mathrm{ey}}$ & 0.2 & 0.0 & \\
\hline Peanut & $19^{\mathrm{a}}$ & 0.9 & $22 \cdot 1^{\mathrm{bc}}$ & 8.5 & $62 \cdot 0^{\mathrm{gh}}$ & 9.5 & $24 \cdot 1^{\mathrm{e}}$ & 1.7 & $4 \cdot 2^{\text {cde }}$ & 0.7 & $20.0^{\mathrm{d}}$ & 0.7 & $2 \cdot 9^{\mathrm{dy}}$ & 0.1 & $2 \cdot 2^{\mathrm{cz}}$ & 0.1 & $6 \cdot 0^{\mathrm{ax}}$ & 0.2 \\
\hline Pole sitao & $4 \cdot 2^{c}$ & 0.1 & $11.5^{\mathrm{e}}$ & 4.4 & $75 \cdot 8^{\text {efg }}$ & $4 \cdot 6$ & $35 \cdot 0^{\mathrm{b}}$ & 0.2 & $5 \cdot 5^{\mathrm{b}}$ & 0.3 & $29.5^{\mathrm{b}}$ & 0.6 & $5 \cdot 6^{\mathrm{ax}}$ & 0.5 & $1.9^{\mathrm{dx}}$ & 0.1 & $0.7^{\mathrm{hz}}$ & 0.0 \\
\hline Cowpea & $3 \cdot 2^{d}$ & 0.1 & $22 \cdot 3^{\mathrm{c}}$ & 3.9 & $67 \cdot 0^{\mathrm{g}}$ & $4 \cdot 1$ & $34.0^{\mathrm{b}}$ & 0.6 & $4 \cdot 0^{\mathrm{de}}$ & 0.2 & $29 \cdot 8^{\mathrm{b}}$ & 0.6 & $4 \cdot 8^{\text {by }}$ & 0.1 & $2 \cdot 1^{\mathrm{cdz}}$ & 0 & $5 \cdot 4^{\mathrm{bx}}$ & 0.2 \\
\hline Chickpea & $2 \cdot 3^{f}$ & 0.1 & $20 \cdot 7^{\mathrm{c}}$ & 0.1 & $69 \cdot 5^{\mathrm{g}}$ & 0.3 & $26 \cdot 2^{\mathrm{e}}$ & 0.7 & $1 \cdot 3^{\mathrm{g}}$ & 0.1 & $24.9^{d}$ & 0.6 & $2 \cdot 8^{\mathrm{dx}}$ & 0.2 & $0.6^{\text {fghz }}$ & 0.2 & $0.9^{\mathrm{fy}}$ & 0.0 \\
\hline Green pea & $1 \cdot 0^{\mathrm{h}}$ & 0.1 & $21.5^{\mathrm{cd}}$ & 0.1 & $69 \cdot 4^{\mathrm{g}}$ & 0.3 & $29.7^{d}$ & 0.9 & $2 \cdot 1^{\dagger}$ & 0.1 & $27 \cdot 6^{\mathrm{c}}$ & 0.6 & $1 \cdot 9^{f x}$ & 0.1 & $0 \cdot 7^{\mathrm{fy}}$ & 0.1 & $0.6^{\text {iy }}$ & 0.0 \\
\hline Lima bean & $2 \cdot 0^{\mathrm{g}}$ & 0.1 & $23 \cdot 3^{\mathrm{c}}$ & 1.9 & $60 \cdot 1^{\mathrm{h}}$ & 2.5 & $20.9^{f}$ & 0.1 & $3.7^{e}$ & 0.1 & $17 \cdot 7^{\mathrm{e}}$ & 0.2 & $2 \cdot 0^{f x}$ & 0.1 & $0.6^{9 y}$ & 0.0 & $0.1^{\mathrm{kz}}$ & 0.1 \\
\hline Kidney bean & $2 \cdot 5^{\text {ef }}$ & 0.3 & $28 \cdot 3^{\mathrm{b}}$ & 0.0 & $60 \cdot 4^{\mathrm{h}}$ & 0.3 & $29.8^{d}$ & 0.3 & $0.4^{\mathrm{h}}$ & 0.1 & $29 \cdot 4^{\mathrm{b}}$ & 1.8 & $0.4^{\mathrm{hz}}$ & 0.2 & $7 \cdot 2^{\mathrm{ax}}$ & 1.5 & $3 \cdot 6^{\mathrm{cy}}$ & 0.7 \\
\hline Pigeon pea & $1 \cdot 3^{\mathrm{h}}$ & 0.2 & $24 \cdot 5^{\mathrm{c}}$ & 0.1 & $63 \cdot 2^{h}$ & 0.4 & $21 \cdot 8^{f}$ & $1 \cdot 1$ & $2 \cdot 4^{\dagger}$ & 0.2 & $19 \cdot 4^{d}$ & 0.6 & 0.0 & & $3 \cdot 3^{b x}$ & 0.2 & $1.5^{\mathrm{ey}}$ & 0.1 \\
\hline
\end{tabular}

$\mathrm{CHO}$, carbohydrates

${ }^{a-1}$ Mean values within a column with unlike superscript letters were significantly different $(P<0.05)$

${ }^{x y z}$ For SCFA mean values within a row with unlike superscript letters were significantly different $(P<0.05)$.

${ }^{*}$ Calculated by difference (100 - (ash, moisture, fat, protein)).

† SCFA (mmol/g fibre isolate).

\section{Reference}

1. Aida C. Mallillin, et al. (2008) Dietary fibre and fermentability characteristics of root crops and legumes. British Journal of Nutrition, doi:10.1017/S000711450891151X 